\title{
Scenario-Based Design for Multiple Microgrids with High DG Penetration Considering Uncertainty on Demand and Generation Side
}

\author{
Hossein Soory ${ }^{1}$, Alireza Sedaghati ${ }^{*}$
}

\author{
Power Engineering Department, Faculty of Electrical and Computer Engineering, Shahabdanesh Uniy $\lambda$, \\ Pardisan, Qom, P. O. B. 3749658479, Iran \\ * Corresponding author, e-mail: ars@shdu.ac.ir
}

Received: 09 December 2018, Accepted: 20 February 2019, Published online: 09 April 2019

\section{Abstract}

In this paper a new scenario-based approach is proposed to design optimized load consumption and renewable DGs generation. The proposed method is used to determ,
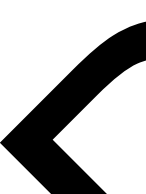
and location of renewable and controlable distributed generation resoyrer optimal location to cluster the traditional distribution network into a set of interconnected microgrig decrease the total design costs including investment and operation cd energy not supplied cost. Different considered objective functions hay een modeled usi weighted coefficients method as a singleobjective nonlinear mixed integer problem. In addition, the uncertaint the problem j ut parameters is modeled using scenario generation method, and in order to decrease the comp tional burden program execution speed, the backward

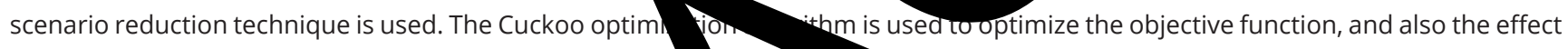
of optimization coefficients on the design problem and t robustroposed algorithm are investigated using sensitivity analysis. Finally, the efficiency and performance of the pr method are evaluated on the standard 33-bus network and the results show that the proposed method jerds multiple microgrid, reliability, ren

\section{Introduction}

Conventional tribution syste (DS) have been designed to have a acity adequate to mo demand required at any were flows in one direction to the consume vrrently DS is being impacted by an increasing installatio ferent typ of distributed energy resources such hotoy and wind turbines.as well as a wide ange of $\mathrm{ng}$ demandside management (DSM) red e. battery storage and electric vehicles (EVs) [1]. roday, andribution network designers are being faced to issues including significant load growth, demand growth, limited fossil sources, distribution network low eliability due their radiality, and customers' geographic expansion [2]. On the one hand, due to environmental policies, new challenges have emerged in utilizing power generation sources for power system designers, and power system investors have little desire to construct fossil fuel plants [3]. Furthermore, the connection of distributed generation resources to the current distribution networks has not provided the technical and economic benefits for investors, while it was expected that increasing the penetration rate of distributed generation sources would increase the power quality, but due to the power fluctuations caused by voltage and frequency difference in renewable energy resources, the desired results have not been obtained [4].

The increasing penetration of renewable energy resources (RER) and their stochastic behaviors on the one hand and also, sharp fluctuations of electricity prices on the other hand have created substantial challenges for optimal operation of microgrids (MG) [5]. Therefore, the appropriate solution to solve the above-mentioned problems is to create small independent grids or the so-called "microgrids". Microgrid, which is composed of integrating several distributed resources at low and medium voltage levels, is one of the most suitable ways to generate 
power in future distribution networks. According to the definition of the American Energy Agency [6], various references have addressed the microgrids design issues with different perspectives. In [7], an overall overview of the various methods used to design microgrids is presented.

Reference [8] proposed an optimal method in order to design multiple microgrids with respect to the distribution system reliability and security. The main purpose of this paper is to cluster distribution networks into a set of microgrids with consideration of network reliability [9]. In [1], linearization system can be implemented mainly using three techniques, such as sampled sensitivity, Zbus matrix and analytically. The sampled based technique is easier to implement but processing time is slow, while the Zbus matrix method is the more precise linear model, but it is unable to capture non-linear behavior. The analytical method is challenging to implement but its processing time is very quick ensuring the maximum possible precision of linearization. A multi-objective economic approach is proposed to design a unit microgrid taking into account the probabilistic characteristics of renewable distributed generations. Desig self-repair microgrids to tolerate contingencies in dist tion network is examined in [10]. In this reference, the dis bution network is partitioned into a set of microorids aimin to minimize system load shedding unde canter ditions and operating cost in fault conditions

Reference [11] is concerned bution networks in the form of increasing the overall reliabl gainst natural and rare accidents. per presents $d_{1}$ val operational strategies dur severe ts with high amain and low probability yich is becam erational by zoning distribution work into a cluster or rogrids.

Voltag se is one main concerns that can limit the allow venety of DG. During high power generation of DO ght load ods (extreme state), there anst of $\mathrm{r}$ se power flow, and therefore voltage se, in thoution feeder. The conventional volta daculation devices such as on-load tap changers cs) arencapable of treating these issues comtely without aproper coordination with DG. An optim. ion model is proposed to determine the optimal DG inver oversize for voltage regulation in the distribution system [12]. Reference [13] proposed an optimal model to design microgrids, taking into account the uncertainty of renewable distributed generations such as wind turbines and photovoltaic systems. In this paper, the objective function involves the installation and operating costs of generation units and the energy storage resources, while the reliability and environmental aspects are not considered. In [14], the microgrid is designed using Graph theories. In this paper, the grid structure design investigated using the graphs partitioning regard to system reliability. In $[15,16]$ generations are optimally integrated at the dium voltage site of distribution network, the ne k adequacy has been investigated us rithm. In addition, in [17], clustered aiming to im and tele-communica

In this paper, a MG consist of dispatchable arces including WT and $\mathrm{V}$ and $\mathrm{d}$ chable resources including Phospho Fuel Cell FC), Micro-gas Turbine (MT and electrical storage as attery Energy Storage Sy $m$ (BESS) is in stigated to minimize operation cost and missions [18]. A ough the design of multiple microgrid reported in mentioned studies, the design of microg ans all economic, technical, environental and renrability aspects regarding the uncertainty if design parameters is not reported in previous ferences. In addition, with respect to the problem type, which is a non-linear and non-convex problem, it is much more desired to employ novel and improved optimization lgorithms to achieve more optimal results. To this end, in this paper an optimal and comprehensive approach is proposed with regard to the economical, technical, environmental, and reliability aspects aiming to introduce an economic and reliable structure considering the power exchange between microgrids and upstream network in order to increase the system reliability and reduce the load shedding in islanding mode for multiple microgrids.

The above calculations have been evaluated under the probabilistic environment in which the scenario generation method is used to cover the problem uncertainty. The proposed objective function is optimized using a new intelligent algorithm named Cuckoo optimization algorithm, with the consideration of various network operating and technical constraints.

Fig. 1 shows the microgrids design concepts including DGs placement and microgrids bounding in distribution network.

In general, the paper contributions are as follows:

- Various types of di stributed generation resources are designed optimally and coordinated.

- The microgrids electrical boundaries are determined using switch placement. 


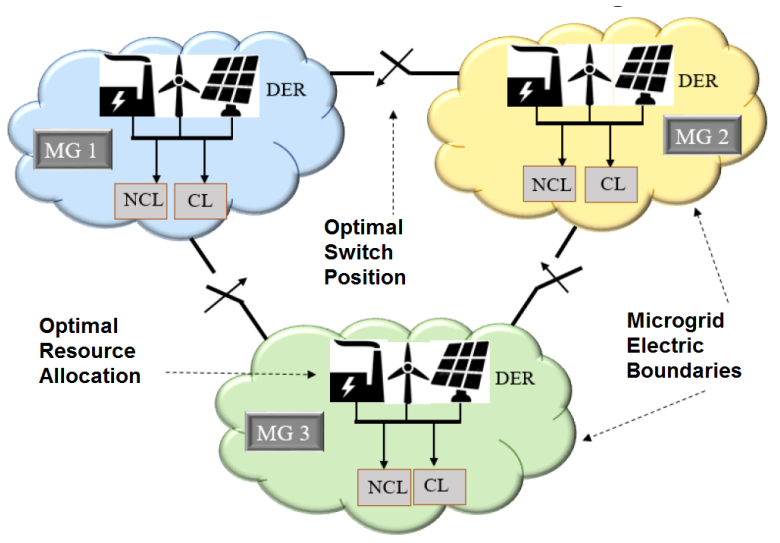

Fig. 1 The concepts of method proposed to design microgrids

- Cost, reliability and air pollution are considered at the same time.

- The load and production uncertainty are modeled using scenario generation method.

A new optimization algorithm called Cuckoo algorithm is employed. In Section 2, the probabilistic mode of resources as well as the network load consumption sented. Section 3 describes the proposed methodolog formulation. The algorithm used for optimization is reporte Section 4. Section 5 provides and eva results and the overall conclusion

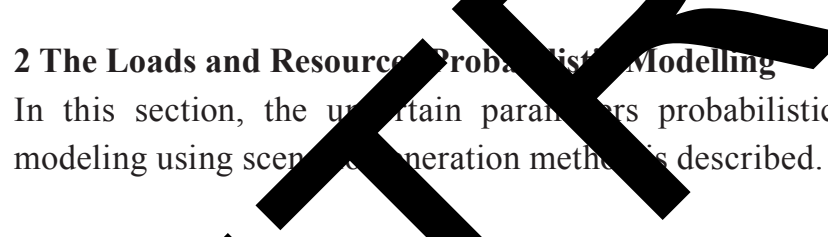

\subsection{Consump in Load}

The distrib on system demand de s to a large extent on humar avities an time factor. In the theory of probability, stoch behavior of parameters could be represented by ability den function proportional to their

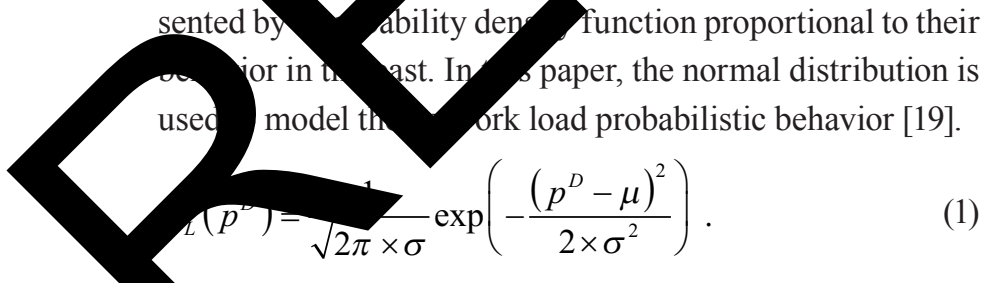

Where $\mu$ represents the load average value, $\sigma$ is the standa deviation of network load probability distribution, and $p^{D}$ depicts the microgrids power consumption, where a number is obtained for $F_{L}\left(p^{D}\right)$ for each $p^{D}$. It is worth to notice that the above relation shows the annual peak load.

\subsection{Wind Turbine}

The wind speed is continuously changing and the exact speed could not be determined for a particular geographic area. Referring to the prior data of wind speed imarticular region, we could obtain a suitable tion to determine wind speed distribut In this paper, the Weibull distribution for is used to model the wind variations [20 general function of Weibull

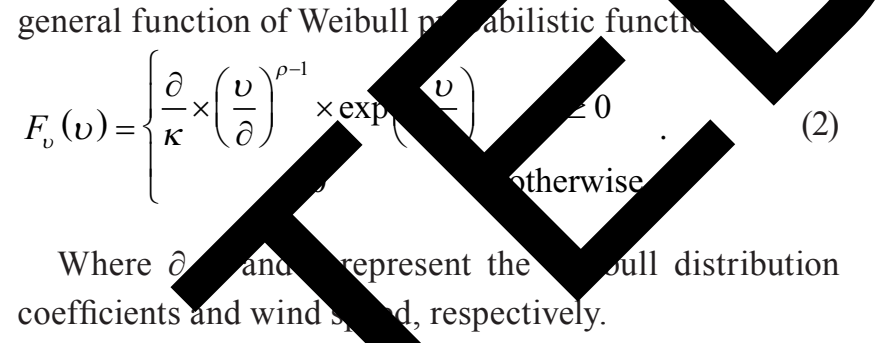

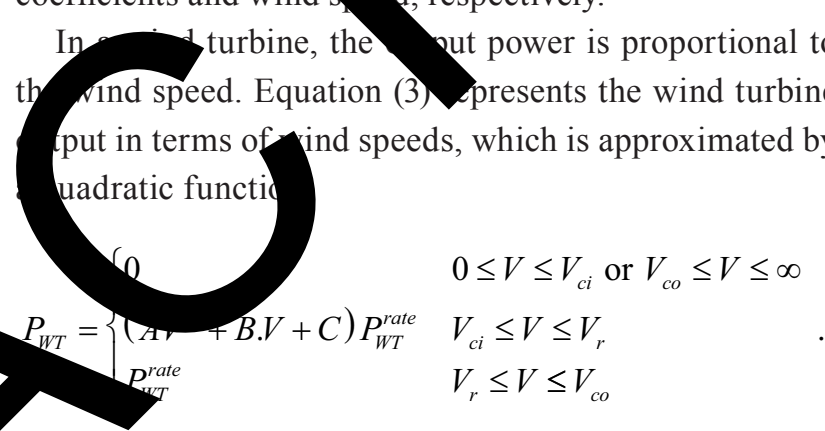

Where the variables $V_{c o}, V_{r}$ and $V_{c i}$ represent the cutspeed, rated speed and connection speed of wind turbine, respectively, and $P_{W T}^{\text {rate }}$ depicts the wind turbine rated power under its rated speed.

\subsection{Solar Cell}

The solar radiation rate and the air temperature of the ambient where the solar cells are located cause the variability of the generation capacity of solar power resources. In this paper, a beta probability distribution function is used to show the sunlight variation curve [20], in which the general relation between beta distribution and the shape parameters $\Omega$ and $\varphi$ are presented in Eq. (4).

$F_{s}(S)=\left\{\begin{array}{cc}\frac{\Gamma(\xi+\varphi)}{\Gamma(\xi)+\Gamma(\varphi)} \times S^{\xi} \times(1-S)^{\varphi}, & 0 \leq S \leq 1 \\ 0 & \text { otherwise }\end{array}\right.$

$P_{P V}=P_{S T G} \times \frac{G_{I N G}}{G_{S T G}} \times\left(1+k\left(T_{C}-T_{r e f}\right)\right)$.

Equation (5) shows the power generated by solar modules under different environmental conditions. The solar 
module output power is determined according to the above relation. In this relation, $k$ is the thermal coefficient and $P_{S T G}$ is the power generated under the rated conditions. $G_{I N G}, G_{S T G}$ are solar radiations in standard and normal conditions, and $T_{C}$ and $T_{r e f}$ are the temperature around the cell and the standard temperature.

\subsection{Combined Heat and Power Generation resources (CHP)}

The combined heat and power sources are known as one of the most economical and most efficient power generation sources, which are capable to simultaneously generate electrical power and heat. These resources are made in three types, whereas only the electrical power type is used in this paper. The fuel cost of these units is calculated using Eq. (6) as shown in [21]. In this relation, $\alpha$, $\beta$ and $\gamma$ are the generation cost coefficients of combined heat and power generation units, and PCHP is the source generated power.

$P_{C H P}(G)=\alpha+\beta \cdot P_{C H P}+\gamma \cdot P_{C H P}^{2}$.

$$
\begin{aligned}
& S=\phi_{d} \times \phi_{w} \times \phi_{s} \\
& \sum_{S \in N_{s}} \psi_{d}+\psi_{w}+\psi_{s}=1 .
\end{aligned}
$$

In the above equation, $\psi_{w}, \psi_{d}$ and $\psi_{s}$ repres ability of possible load conditions, wind modules generations, respectively. In addit $C_{w}, C_{d}$ and $C_{s}$ depict the numerical amount of aes ext d from probability distribution for loa generations, respectively, generated scenarios.

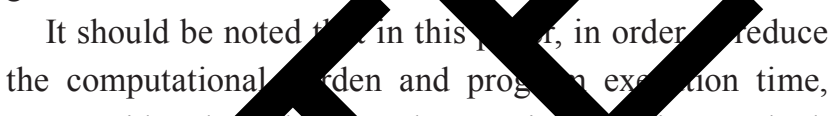
we considered ba ard scenario method. The scenario reduction is qy to optimally choose useful scenario s set of gene scenarios, which reduces the orithm execution time and so greatly decreases the pro em computation l complexity. The backward scenar reduction meth steps are described below:

- vnerate distan matrix between scenarios as $C\left(S, S^{\prime}\right)$

- So firsta ario using Eq. (12)

Select next scenario and add it to the previous scenario

\subsection{Generation a nd Reduction of Scenarios}

Due to the uncertainties in microgrids consumption loa and power generation of renewable ener sur such as wind turbines and solar cells, desig microg is with consideration of the uncertainty he ecome a lenge for distribution system/igne o s paper, mo scenario generation meth $1 \mathrm{~s}$ used to or the uncertainty of system design ters. In this ap using the Monte Carlo sin ation m d, a number $d$ stochastic conditions ar enerated for $\mathrm{s}$ v variables based on their probabi distribution functiol od then, the probability of condit is calculated 22]. Equations (7) to (9) sh the ext samples from probabilistic distributions for and generg and solar power, respec-

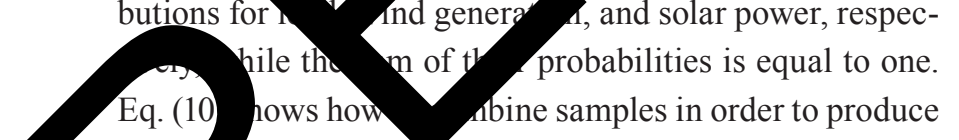
sceng sarthat the sum of the generated scenarios probabi es is alway qual to one as it can be seen in [10].

$$
\begin{aligned}
& =\left\{\left(C_{d}^{1}, \psi_{d}^{1}\right),\left(C_{d}^{2}, \psi_{d}^{2}\right), \ldots,\left(C_{d}^{n}, \psi_{d}^{n}\right)\right\} \\
& \phi_{w}=\left\{\left(C_{w}^{1}, \psi_{w}^{1}\right),\left(C_{w}^{2}, \psi_{w}^{2}\right), \ldots,\left(C_{w}^{n}, \psi_{w}^{n}\right)\right\} \\
& \psi_{w}^{1}+\psi_{w}^{2}+\ldots+\psi_{w}^{n}=1 \\
& \phi_{s}=\left\{\left(C_{s}^{1}, \psi_{s}^{1}\right),\left(C_{s}^{2}, \psi_{s}^{2}\right), \ldots .,\left(C_{s}^{n}, \psi_{s}^{n}\right)\right\} \\
& \psi_{s}^{1}+\psi_{s}^{2}+\ldots+\psi_{s}^{n}=1
\end{aligned}
$$

$S_{n}=\arg \left\{\min _{S^{\prime} \in N_{s}} \sum_{S} \psi_{s} \times C\left(S, S^{\prime \prime}\right)\right\} N_{s}$.

More detailed information on available methods for scenario reduction is provided in [23].

\section{Problem Formulation}

\subsection{Economic assessment}

In this section, we perform an economic assessment for installation of distributed resources and power switches, including investment and operation costs to design microgrids. It is worth noting that proposed method is a static method in which all investments are made in the first year of the design horizon.

\subsubsection{Investment cost}

The investment cost of distributed generation resources and switches involves the installation cost and the costs concerned with the land. Equation (14) presents the costs of distributed generation and switches for all scenarios. 


$$
C_{1}=\sum_{s=1}^{N_{s}}\left(\sum_{i=1}^{N_{D G}} I C_{i, s}^{D G} \times P_{i, s}^{D G} \times \eta_{i, s}^{D G}+\sum_{m=1}^{N_{S W}} I C_{m, s}^{S W} \times \theta_{m, s}^{S W}\right) .
$$

Where $I C_{i, s}^{D G}$ is the sources construction cost, $P_{i, s}^{D G}$ is the amount of sources generated power and $\eta_{i, s}^{D G}$ is a binary variable to install source $i$ in the scenario $s$. In addition, $I C_{m, s}^{S W}$ is the switches installation cost and $\theta_{m, s}^{S W}$ is a binary variable to install switch $m$ in scenarios.

\subsubsection{Repair and Maintenance Cost}

This cost involves the annual costs of the periodic and seasonal repairs associated with installed distributed generation resources and power switches, which is presented by Eq. (15) and is modified using inflation and interest rates as Eq. (16). In Eq. (15), $M C_{i}^{D G}$ is the resources repair and maintenance cost and $P_{i, s}^{D G}$ shows the sources generated power in scenario $s$. In addition, $M C_{m}^{S W}$ is the repair and maintenance cost of installed switches and $\tau_{m, s}^{S W}$ is the switching rate of switch $m$ in scenario $s$. In Eq. (16), $\ln f R$ and $\ln t R$ represent the inflation rate and interest rate in the operation horizon, respectively.

$$
\begin{aligned}
& C_{2}=\sum_{s=1}^{N_{s}}\left(\sum_{i=1}^{N_{D G}} M C_{i}^{D G} \times P_{i, s}^{D G}+\sum_{m=1}^{N_{S W}} M C_{m}^{S W} \times \tau_{m, s}^{S W}\right) \\
& C P V\left(C_{2}\right)=C_{2} \sum_{s=1}^{N_{s}} \sum_{t=1}^{T}\left(\frac{1+\ln f R}{1+\ln t R}\right)^{t}
\end{aligned}
$$

\subsubsection{Operating cost}

The operating cost incly the cost of il fuel unis, such as he combined heat wer generatio vits, which is obtained using E 17 ) in rent operation years, and is modified at different ope $\mathrm{yghorizons}$ as shown in Eq. (18). nese relationships, $\mathrm{O}_{G}$ is generation productio st, $T_{i, s}^{D G}$ he operation time, and $P_{i, s}^{D G}$ represent $i$ mount generated power in scenario $s$.

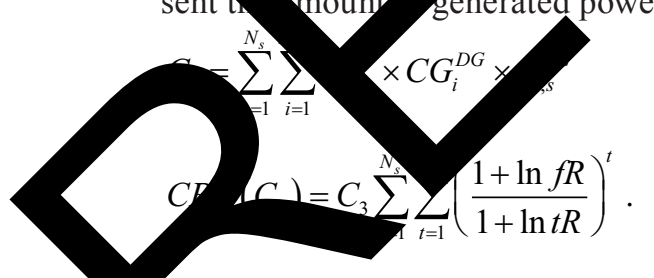

It is worth noting that renewable resources lack the priy fuel cost and their operating costs are considered on. $\mathrm{in}$ terms of maintenance and repair costs.

\subsection{Technical assessment}

This section addresses the technical aspects of microgrid design, including the air pollution cost due to the fossil fuel based distributed generations and the network losses cost.

\subsubsection{Air pollution cost}

According to the Kyoto treaty and the need to reduce greenhouse gases, consideration of this discussion in microgrids design has become an important goal of design-mare distribution networks. In this paper, the tion is modeled according to the Eqs.

$$
C_{4}=\sum_{s=1}^{N_{s}} \sum_{i=1}^{N_{C H P}}\left[\zeta_{i}^{C H P} \exp \left(\delta_{i} \times P_{i, s}^{C H P}\right)\right]
$$

20) [24].

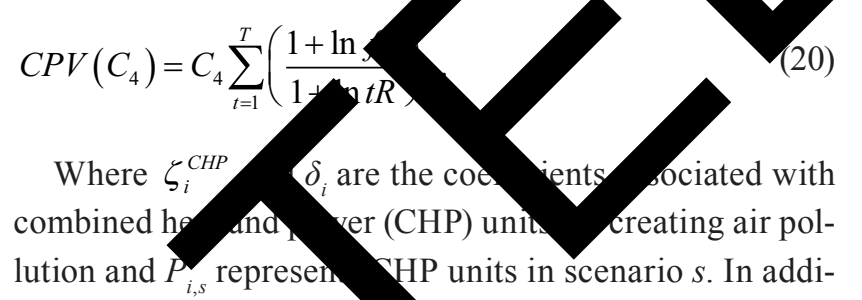

tion. $\longrightarrow$ calculates thent value of cost caused by 2 The loss cos $)$ ork loss is one of the most importwork quanıy. System loss calculation requires running the Gow program on the studied system. In this paper, the backward-forward power flow method has been used as a reliable and suitable method for high-resistivity distribution networks [25]. Due to the problem probabilistic modeling, the system loss is also presented probabilistic using scenario generation method in Eq. (21), and the current cost of loss is calculated using Eq. (22).

$C_{5}=\sum_{s=1}^{N_{s}} \sum_{b=1}^{N_{b}}\left(R_{b} \times I_{b, s}^{2}\right) C_{\text {loss }}$

$C P V\left(C_{5}\right)=C_{5} \sum_{t=1}^{T}\left(\frac{1+\ln f R}{1+\ln t R}\right)^{t}$.

Were $R_{b}$ is the network line resistant, $I_{b, s}^{2}$ is the lines current and $C_{\text {loss }}$ is the dollar per kilowatt cost of energy loss.

\subsection{Reliability Assessment}

One of the most important goals to convert radial distribution networks into a set of interconnected microgrids is the need to increase the distribution networks reliability level. Due to the distribution networks radial structure and the low reliability of these networks, clustering the distribution networks as a set of interconnected microgrids improves the network subscribers' reliability [25]. The proposed method also assesses the distribution system reliability which is discussed in the following. 


\subsubsection{Cost of energy not supplied}

Energy not supplied is one of the most important indicators to evaluate distribution networks reliability, which provides complete information about the behavior and performance of system. This indicator shows the amount of power loss due to the contingencies at network lines, which is calculated annually. It is important to note that this paper assumes that faults only occur at network lines and other network equipment is $100 \%$ reliable. Eq. (23) represents the subscribers interruption cost for different scenarios, whose current cost is calculated using Eq. (24) for different years of operation.

$$
\begin{aligned}
& C_{6}=\sum_{s=1}^{N_{s}}\left\{\begin{array}{l}
\sum_{b=1}^{N_{b}} C_{\text {int }} \times \lambda_{b} \times L_{b} \times P_{b, s}^{D} \times \sum_{r e s=1}^{N_{\text {res }}} P_{s}^{r e s} \times T_{s}^{r e s} \\
+\sum_{r e p=1}^{N_{\text {rep }}} P_{s}^{r e p} \times T_{s}^{r e p}
\end{array}\right\} \\
& C P V\left(C_{6}\right)=C_{6} \sum_{t=1}^{T}\left(\frac{1+\ln f R}{1+\ln t R}\right)^{t} .
\end{aligned}
$$

Where $\lambda_{23}$ is the lines failure rate, $L_{b}$ is the length tribution network lines, PBD is the power amount of $i$ rupted load, and $C_{i n t}$ is the cost of each subscriber outa per kWh. In addition, $P_{s}^{r e s}$ and $T_{s}^{r e s}$ represent the amour of interrupted power due to repairs and entrs

\subsection{Objective function and probK constrai} In general, the objective fun $\mathrm{n}$ co to three pars including economic cost, to rical cost, a liability cost, which are shown using 5 ) to (27). Tho timization aims to minimize thal men costs at the ame time with respect to different syste onstraints. The problem main va including the pla sizing the different 4 s of dist ated generation resources, as well as the op loca of switches in order to determine the

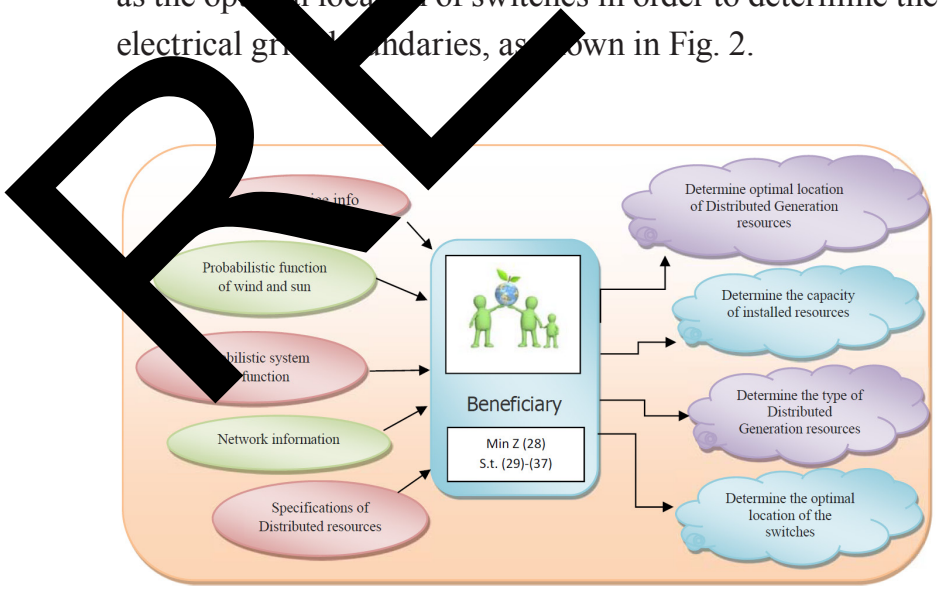

Fig. 2 The decision diagram to design microgrids
The considered objective functions have converted into a single objective function using weighted coefficients method [26].

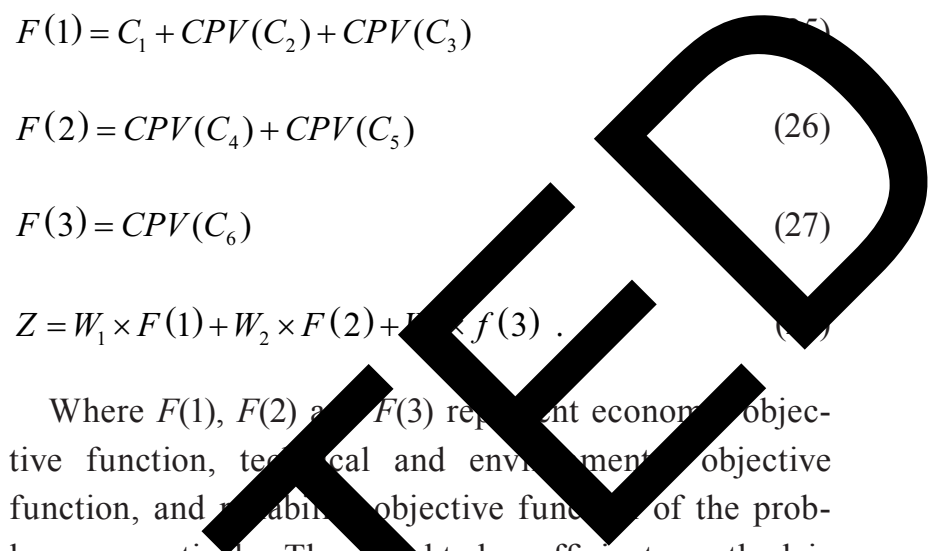
lem, respectively. The hted coefficients method is used to these three ctions to a unit objective fung as shown in Eq. (28).

e optimization poblem is carried out under various op tional and tech al constraints. In this paper, the prob constraints clude the allowed range of buses voltage range of feeders' current, the maxium number or DGs, each source capacity range and the p ilibrium constraint for each microgrid, as well as power row equations, which are formulized using constraints (29) to (34) and Eqs. (35) to (37). Constraint (29) indicates the permitted voltage range for network buses, while in this paper, the allowed range of bus voltages is considered to be between 0.95 and 1.05. In addition, the network lines allowed current is limited using constraint (30). Constraint (31) shows the maximum amount of distributed generation resources to be installed in the distribution network and the constraints (32) to (34) present the allowed range to install CHP units, solar cells and wind turbines. In addition, Eq. (35) imposes the power equilibrium constraint to the created microgrids, which makes the microgrids to be completely independent and stand-alone. Finally, Eqs. (36) and (37) show the power flow equations.

$$
\begin{aligned}
& V_{n, s}^{\min } \leq V_{n, s} \leq V_{n, s}^{\max } \quad \forall s \in N_{s} \\
& I_{b, s} \leq I_{b, s}^{\max } \quad \forall s \in N_{s} \\
& \sum_{i=1}^{N_{D G}} P_{i}^{D G} \leq P^{\max } \quad \forall s \in N_{s} \\
& P_{C H P}^{\min } \leq P_{C H P} \leq P_{C H P}^{\max } \quad \forall s \in N_{s} \\
& P_{P V}^{\min } \leq P_{P V} \leq P_{P V}^{\max } \quad \forall s \in N_{s} \\
& P_{W T}^{\min } \leq P_{W T} \leq P_{W T}^{\max } \quad \forall s \in N_{s}
\end{aligned}
$$


$\sum_{n=1}^{N_{M G}} P_{i, n}^{D G}=\sum_{l=1}^{N_{L}} P_{l}^{D}+\sum_{b=1}^{N_{b}} P_{b}^{\text {loss }}, \quad \forall i$

$-P_{t, s}^{D}+\sum_{i \in N_{D G}} P_{i, t, s,}^{D G}=V_{j, t, s} \sum_{b \in N b} Y_{b} . V_{k, t, s} \cos \left(\delta_{j, t, s}-\delta_{k, t, s}-\theta_{j k}\right)$

$-Q_{t, s}^{D}+\sum_{i \in N_{D G}} Q_{i, t, s,}^{D G}=V_{j, t, s} \sum_{b \in N b} Y_{b} . V_{k, t, s} \sin \left(\delta_{j, t, s}-\delta_{k, t, s}-\theta_{j k}\right)$.

Where $V_{n}$ depicts the bus voltage and $n_{l b}$ shows the current flows through line $b$. In addition, $Y_{b}$ is the line admittance, $\delta_{j}$ and $\delta_{k}$ are the voltage angles of buses $i$ and $j$. $Q, P_{i, t, s}^{D G}$ and $P_{b}$ show the installed DGs generated active and reactive power, the network active and reactive power consumption and loss, respectively [21].

\section{Optimization Algorithm}

According to the problem objective function, which is a non-linear and non-convex model with a large number of binary variables and integers, obviously it is not possible to use mathematical methods to solve the problem. Hence, in this paper, the Cuckoo algorithm is used mize the objective function [26].

Similar to other evolutionary algorithms, the $\mathrm{C}$ foo algorithm begins with a primitive population (a pop tion composed of cuckoos). These of eggs that dump them in the these eggs, which are more have a greater chance to oos, while other eggs dentified a

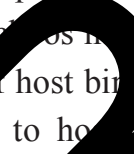
a numb Some o mature cunnhost birds. The amg grown eggs st that region nests are appropr. The eggs in one are able to live and sy ive, the more ats allocated to that area. The re, the location in $m$ the largest number of egg survived he parameter that Cuckoo algorithm inten optin The cuckoos look for the best location to maxin os, form societies and groups. Eacl roup has residence area to live. The best reside aresamong arl groups is the next destination of other ckoos groun. All groups migrate toward best current area. Each group settles in a region close to the current best tion. Considering the number of eggs that each cuckoo lay and cuckoos distance to the current optimal region, some egg laying radius is calculated. Then, cuckoos begin to lay eggs in nests within the egg laying radius. This process continues until the best location for egg laying (most profit) is obtained. This optimal location is where the most numbers of cuckoo come together.
One of the most important problem modeling parts is to determine the input vector for intelligent algorithms variables. The proposed input vector for the Cuckoo algorithm in order to simultaneously model thrand Switch location is shown in Fig. 3. As algorithm input vector consists of two is composed of two separate parts to mal location as well as the opt tributed generation resource shows the location and n

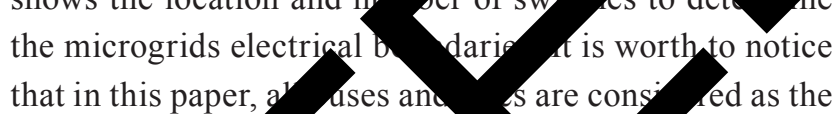
candidate point unstall DGs a

One of $t^{\prime}$ mo mportant fea algorithm is the poss y of global search, in addition to lo when which anding the problem opti$\mathrm{m}$ overall solution. In addit, this algorithm has a less hvergence time han other metaheuristic algorithms. e flowchart ill ated in Fig. 4 depicts the proposed a ithm to desi microgrids under uncertainty using the zation algorithm. ine the opticapach of the disofdition, the sw ed as the witc of the Cuckoo
problem opti-
in addition
tic algorithms.
s the proposed
certainty using ation and Results In this paper, in order to validate the proposed approach performance, a 33-bus standard distribution network is used to implement the proposed method [27]. The costs associated with the construction and operation of resources and switches are obtained from [28-30]. Also, the forecasted load demand and market price profiles are illustrated in Fig. 5 (a) and 5 (b) respectively. The used demand profile is divided into three various periods namely low load period (00:00 AM to 6:00 AM), off-peak period (6:00 AM to 3:00 PM) and peak period (3:00 PM to 12:00 PM) [31]. It is worth noting that the design horizon for the proposed problem is considered to be 10 years.

This paper aims to optimally design the multiple microgrids in an intelligent distribution grid, taking into account the economic, technical and environmental aspects and

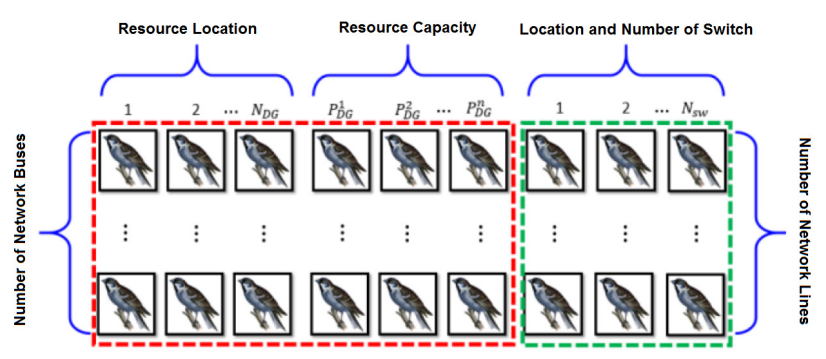

Fig. 3 The proposed input vector for Cuckoo algorithm 


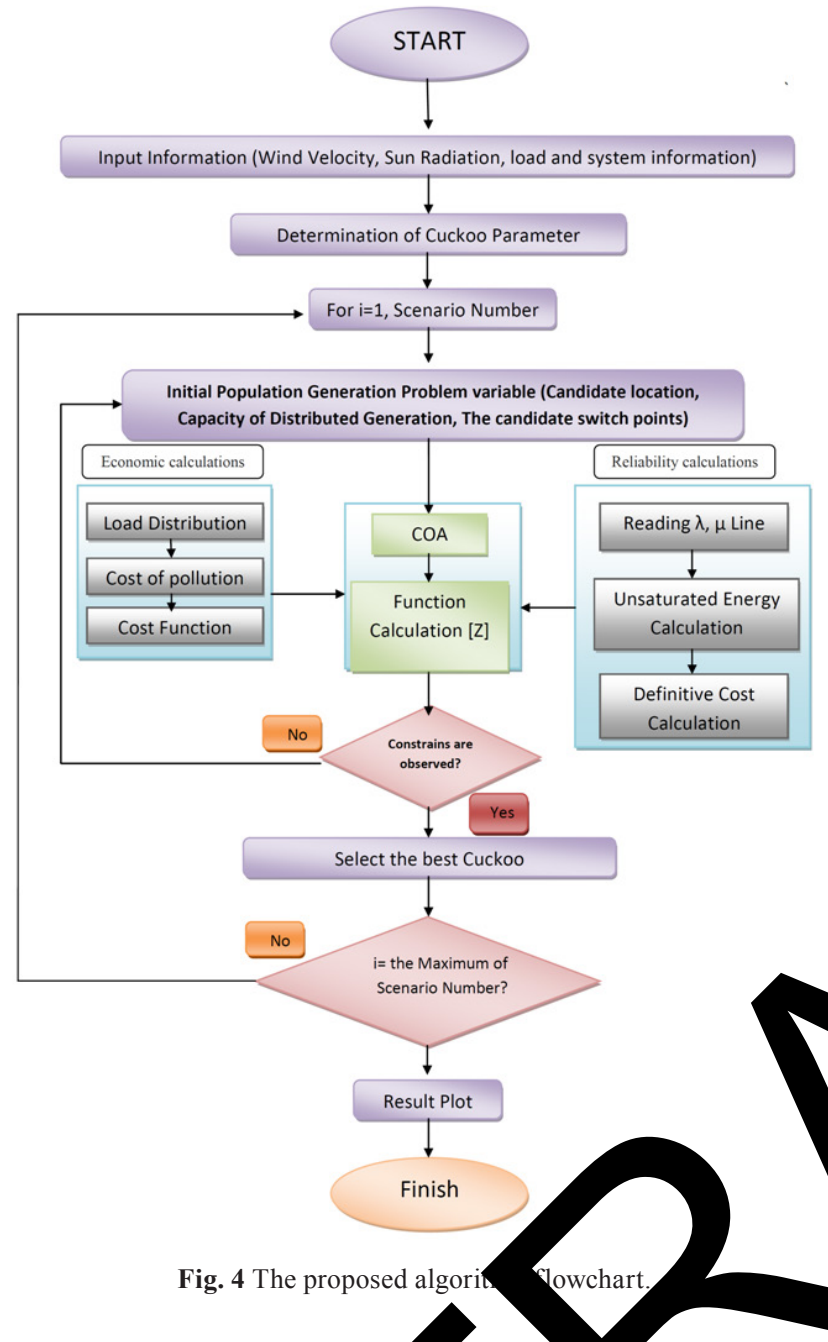

the uncertainty in the dist ation systen sign parameters. The microgrids de oblem is carro us optimal location of rence DQ ources and cartrollable power generatio ources simulto us with switch location in energ stribution network to determine microgriy ectrical andaries. The proposed model is static in ich all estments are made in the first year and determ. of a year onstruct resources is not a variab Uckog mization algorithm has been used as he of the powerful and newest metaheuristic al the optimize the problem objective function. over the uninty, a scenario generation method is ployed to reduce the computational burden and program ru ne using the backward scenario reduction technique.

Ind ding the weighted coefficients in the problem objective function depends on the network designers' policies and strategies, and depending on their goals and priorities, they could have different values between zero and one. In this paper, sensitivity analysis is used to determine the exact impact of optimization coefficients and weighted coefficients on the intended problem.

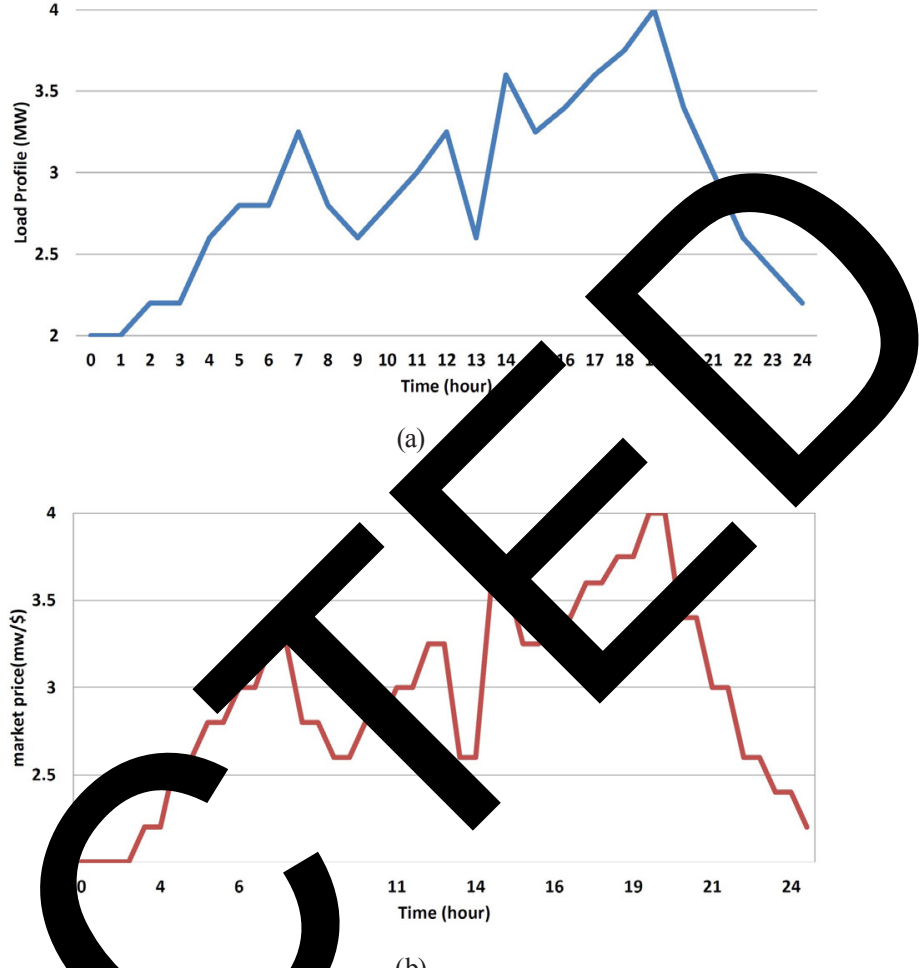

(b)

Fig. 5 (a) aily load demand (b) electricity price curves. hows the results obtained for optimal placement ad sizing of DG resources as well as the switch location for microgrids boundaries based on the obtained average values from probability distribution. According to Table 1, three microgrids are selected for this particular system, and three DG resources are allocated to each microgrid. Three types of DG power resources include solar cells, wind turbines and CHP units are considered for each microgrid.

In addition, Table 2 shows different objective functions optimal values for three different modes of DG resources penetration index (PI). The resources penetration rate is defined as the ratio of the resources installed capacity to the network consumption. According to Table 2, it is clear that with increased penetration of distributed generation resources, the investment and operational costs may increase, while this may improve the technical indicators and system reliability.

Fig. 6 (a) shows the network voltage profile in the both traditional and the multiple microgrids based structures.

Table 1 Optimal placement of distributed generation source

\begin{tabular}{lcc}
\hline Kind of source & Location (Bas) & Capacity (KW) \\
\hline $\begin{array}{l}\text { Sources of electricity and } \\
\text { heat production }\end{array}$ & $12,19,33$ & $200,150,175$ \\
Wind Turbine & $4,9,30$ & $125,100,75$ \\
Solar Cell & $17,24,27$ & $50,100,75$ \\
The switch & $1,6,25$ & -- \\
\hline
\end{tabular}


Table 2 Problem objective function for microgrid design

\begin{tabular}{lccc}
\hline \multirow{2}{*}{ objective functions } & \multicolumn{3}{c}{ objective functions Amounts (\$) } \\
& $\mathrm{PI}=100 \%$ & $\mathrm{PI}=75 \%$ & $\mathrm{PI}=50 \%$ \\
\hline Investment cost & 2.25846 & 1.17388 & 8.12694 \\
maintenance cost & 3.8617 & 8.5792 & 7.4648 \\
Cost of operation & 5.8977 & 3.6619 & 4.5091 \\
Cost of air pollution & 664.7 & 1913.7 & 2846.3 \\
The cost of casualties & 6.1084 & 6.3198 & 3.5297 \\
Cost of reliability & 4.591 & 6.6425 & 1.8846 \\
Total cost & 9.45786 & 2.41335 & 5.394405 \\
\hline
\end{tabular}

According to Fig. 6 (a), the voltage variations of different network buses are much less than that of the traditional one and the voltage level is under different loadings within its allowed range, thus, the system power quality has been improved. Therefore, the proposed method increased the voltage stability margin of the distribution network and prevented the voltage collapse phenomenon in the distribution network.

In addition, Fig. 6 (b) depicts the network loss diagram in two conventional and restructured conditions. According to Fig. 6 (b), in the case where the diribution network is designed as a set of interconnected grids, the system loss is greatly reduced, which imp yes the system technical specifications such as reducing

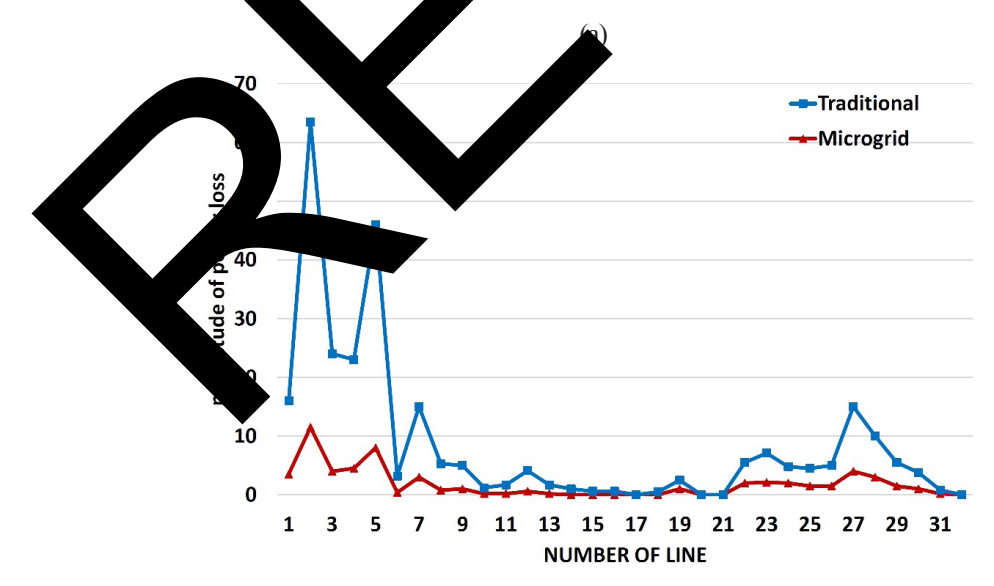

(b)

Fig. 6 (a) Network voltage profile (b) System total loss current passing through lines, and thus, reducing investment costs in distribution feeders, as well as postponing the distribution network upgrade. Reducing the system loss may increase the distribution lines equir life span and decrease the distribution system

Fig. 7 shows a single-line diagram of distribution network which the proposed thod is implemented on. According to Fig. 7 netwo is divided into three interconnected $\mathrm{m}$ grid could be available fo owners. In addition, th network in the cas operate indepe uted genera
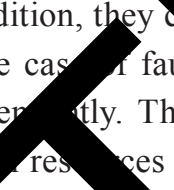
ferent 0 rids, while e capacity is determined ng the proposed algorithm.

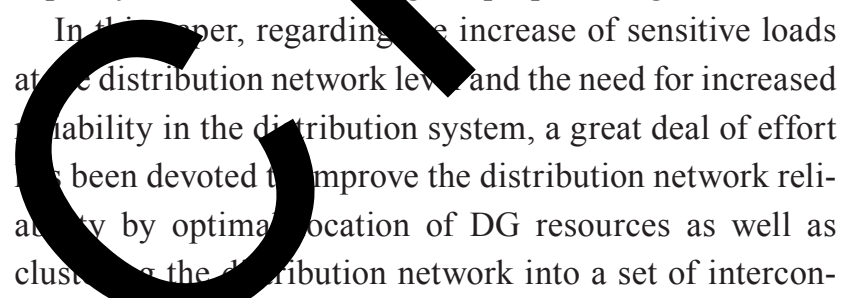
nected microgrids. By changing the weighted coefficients arresponding to the system loss function from 0 to 1, various degrees of system loss could be obtained, which depends on the operational policies and distribution network planners decisions. The exact value of weighted coefficients and their effects on the design problem is determined using sensitivity analysis.

In this study, the energy not supplied index is used as an indicator to evaluate the system reliability. Fig. 8 shows the amount of network energy not supplied in microgrid-based and conventional conditions for different design years. As can be seen, in the case where the distribution networks are designed as a set of multiple microgrids, the amount of energy not supplied significantly reduced due to the splitting the distribution network into smaller parts. It is worth to notice that the reliability assessment is carried out under uncertainty conditions.

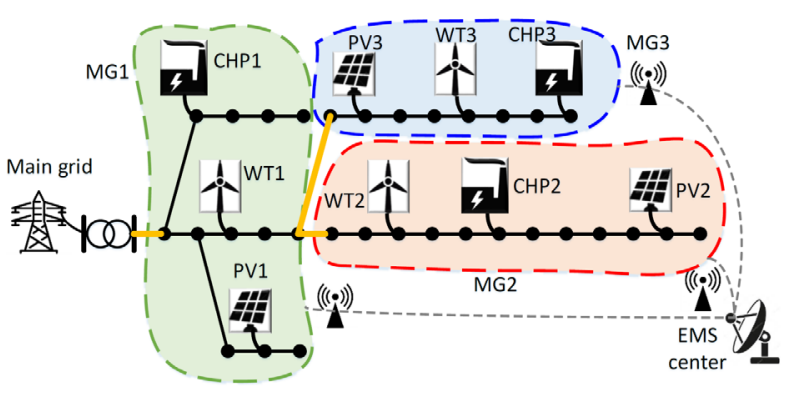

Fig. 7 DG optimal location and multiple microgrids boundaries 


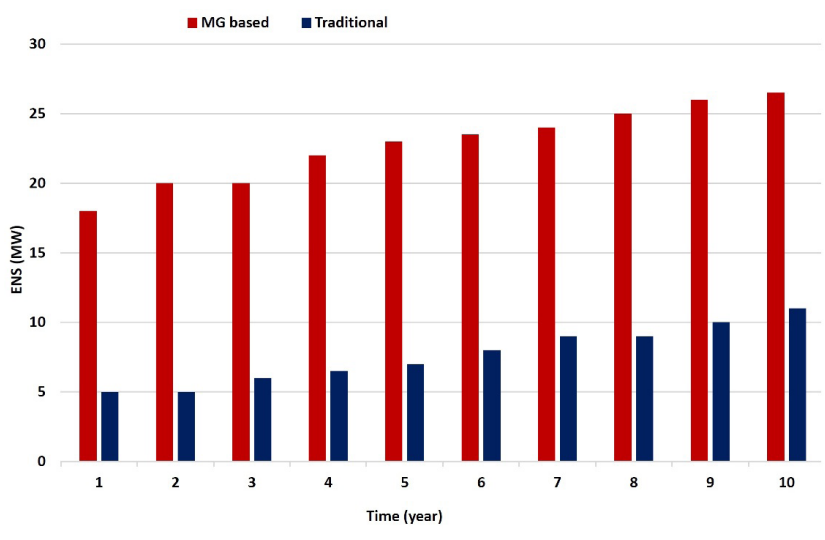

Fig. 8 Energy not supplied values along design horizon

Fig. 9 (a) shows probability diagram of the system energy not supplied index for different scenarios. In distribution networks reliability assessment, the failure rate and repair rate are assigned to each network line, and then, the reliability indicators are calculated based on the faults occurred on each feeder. Fig. 9 (b) shows the probability distribution of the air pollution caused by fossil fuel sources. In addition, Fig. 9 (c) shows the probability distribution of microg design total cost including the investment cost of resou and switches, operating cost, loss and air pollution costs well as energy not supplied cost for different scenarios.

To illustrate the overall design cost that is extracted from probabilis from scenarios is used, which in ble condition for microgrids lem probabilistic modelin

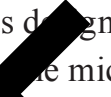
ertain micr against system variab extainty, and
method shows morosed in different op ing condition since the renewable resources po generation depend these resources environy al condis, scheduling these resources based or aver value obtained from the probabilistic distribu ake the no ork design robust against ations. his increa microgrids reliability in both grid- masted and islanding modes.

onsiderny different solutions are obtained for each nario in scenario-based planning, selection of a single sol among the obtained solutions has become one of the da sion makers' challenges. In practice, the average or expected value obtained from the probabilistic distribution of uncertain variables is used for the distribution system planning, which has the highest probability to other scenarios.

However, in recent years various methods are proposed to reduce the risk of costly scenarios [12]. In this

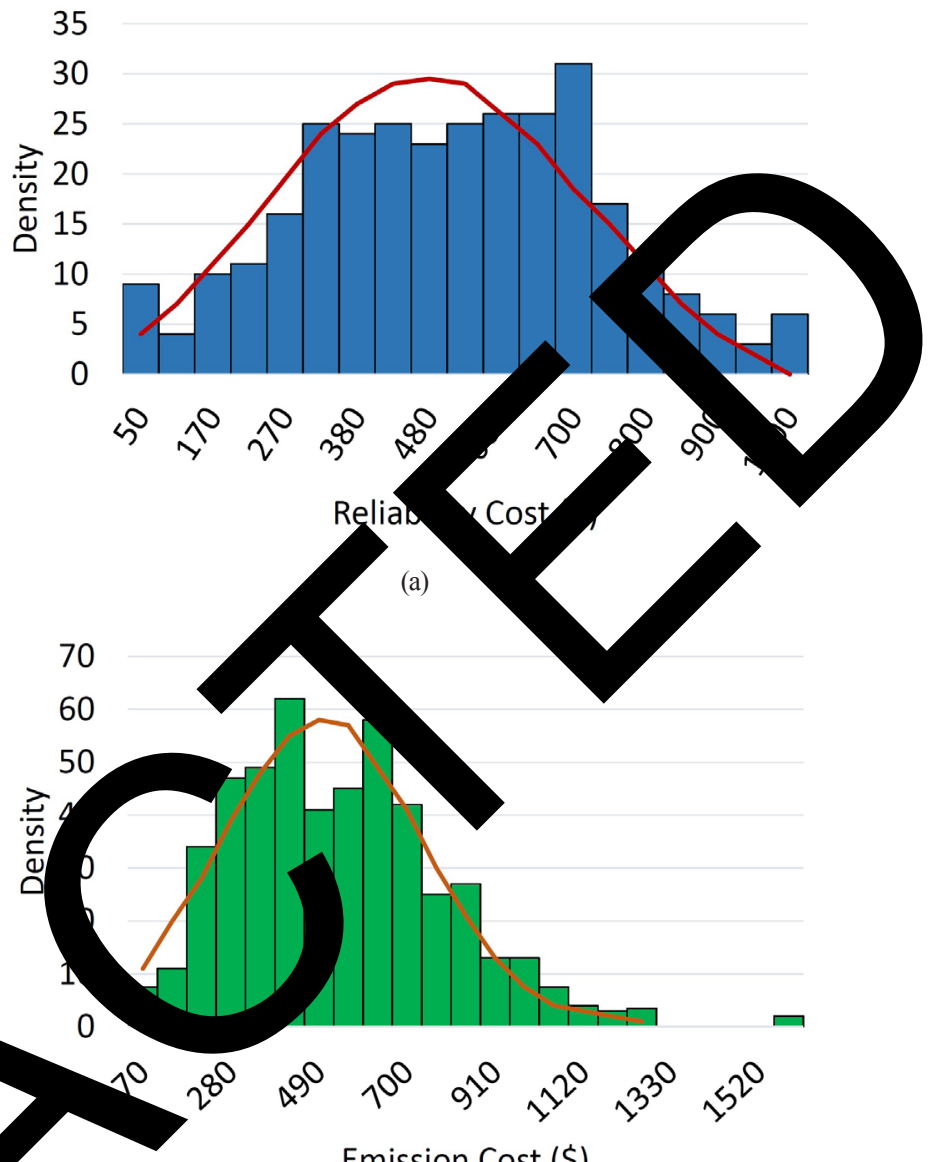

Emission Cost (\$)

(b)

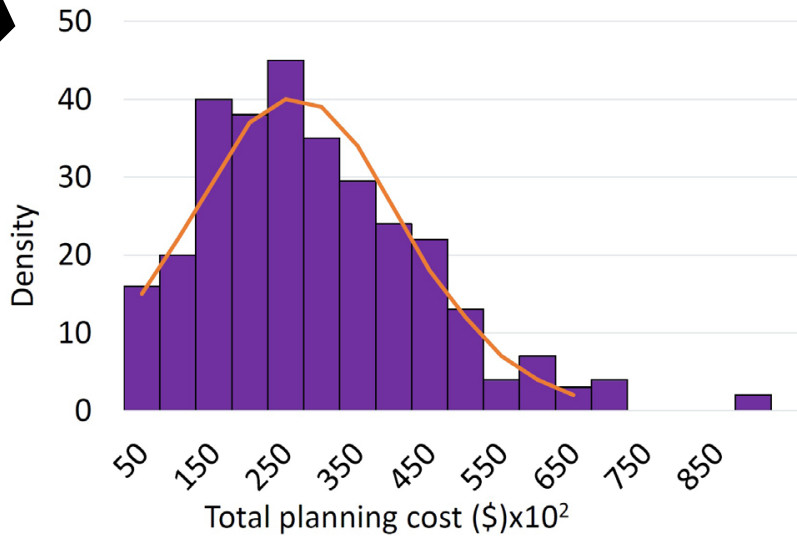

(c)

Fig. 9 (a) The probability distribution of energy not supplied.

(b) The probability distribution of total design cost. (c) The probability distribution of microgrids design total cost.

paper, we used the sensitivity analysis method to examine the effect of weighted coefficients on system design quantity. In this method, the problem is performed for various values that weighted coefficients could have, and the best design in terms of obtained quantities is the optimized weighted coefficient. 
Table 3 shows the impact of weighted coefficients different values on the total cost of the network design. According to Table 3, taking into account all the coefficients may reduce the system total cost. Therefore, designing the microgrids with consideration of all economic, technical and environmental aspects as well as reliability may reduce the network design total cost, while all the three coefficients are considered equal to 1 in this paper. In addition, Table 3 shows the results obtained from sensitivity analysis of weighted coefficients on microgrids boundaries. As is shown, as the network reliability weight is higher the microgrid number increases, while its ultimate value in this particular network is equal to 6 . In addition, as the economical part weight is higher, the microgrids numbers reduces toward a single microgrid, in other words, small microgrids are more reliable and large microgrids are more economic.

In order to illustrate the efficiency and performance of the optimization algorithm used in solving the proposed problem, the Cuckoo algorithm convergence curve is compared with other conventional optimization algorithms (Genetic Algorithm and Particle Swarm Optim tion Algorithm) in Fig. 10. As can be seen, the p algorithm has a remarkable superiority to the othe wo algorithms in terms of convergence speed and obta

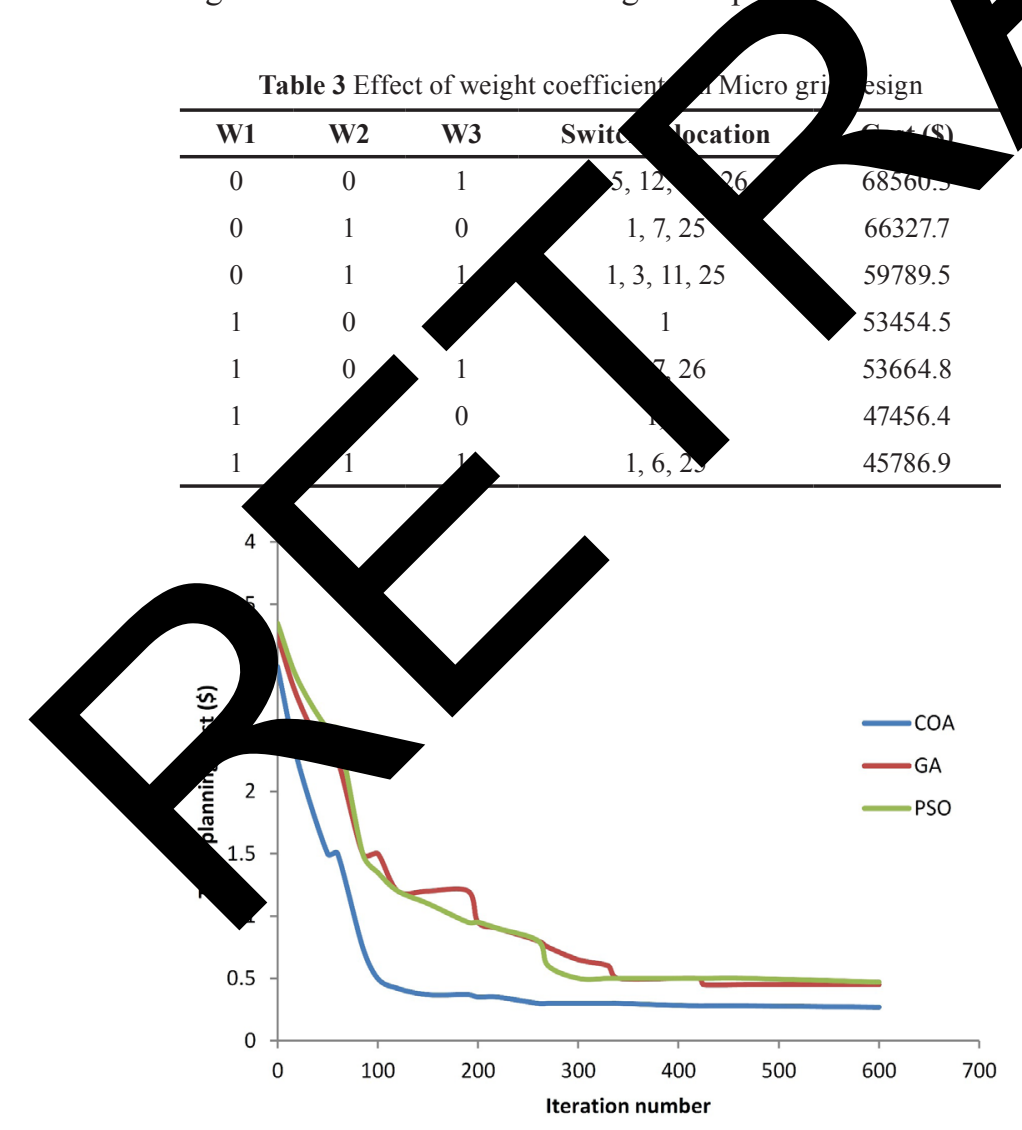

Fig. 10 The proposed algorithm convergence curve values. This is due to the two local and global explorers in cuckoo optimization algorithm which achieve the more desired value in less time.

It is worth to notice that in the scenario-based a mach, selecting the final scenario among the ios is an important issue. In general risk-neutral and risk-taking strategies for this problem. The risk-taking ategy se the lowest possible cost, so that it choos ne scenario the the lowest cost. In contra a risk ar strateg to reduce the system tech risl which may increase the design costs. Th are, in aking stra the scenario with the est cost (the h exp network) is chosen as ult the risk-neutral strategy is the intersti tate of the two mentioned strategies selects the mabable scenario as the final ario. In this paper, we u. 2 ed a risk-neutral strategy find the final serario (single solution). In general, the conomic and reliability assessments ro ts show that a bugh investment costs are increased in the conventional distribution network is splitted into a set of multiple networks, the decrease of reliabilrechnical costs due to the loss reduction and voltage profile improvement is far greater which reduces the entire system cost. According to the obtained results, the best way to design microgrids is to consider three different system aspects at the same time, which imposes the least cost to the distribution system designers. Depending on the planners and grid owners' risk-taking or risk-averse policies, different conditions of weighted coefficients could be selected regarding their needs and the microgrid characteristics.

\section{Conclusion}

In this paper, a non-linear mixed integer planning model is proposed to economic and reliable design of multiple microgrids taking into account the uncertainties in load consumption and renewable resources power generation. The proposed method is used to determine the location, number, type and capacity of renewable and controllable DGs as well as to determine the microgrids electrical boundaries in intelligent distribution networks. In this paper, the scenario generation method is utilized to cover the system parameters uncertainties and the backward scenario reduction method is used to reduce the scenarios in order to increase the computational speed. The results show that clustering the distribution network into a set of interconnected microcircuits improves the economic, technical and reliability specifications of the distribution 
network. In this study, the sensitivity analysis has been used to illustrate the effect of weighted coefficients on the microgrid design. The results show that by changing the different functions weighted coefficients, the obtained structure for microgrids may be extensively changed, while the optimal selection of these coefficients depends

\section{References}

[1] Uddin, S. "Distribution system energy management through capacity constrained optimzation", PhD Thesis, School of Information Technology and Electrical Engineering, The University of Queensland, 2018.

https://doi.org/10.14264/uq1.2018.470

[2] Georgilakis, P. S., Hatziargyriou, N. D. "A review of power distribution planning in the modern power systems era: Models, methods and future research", Electric Power Systems Research, 121, pp. $89-100,2015$.

https://doi.org/10.1016/j.epsr.2014.12.010

[3] Ruester, S., Schwenen, S., Batlle, C., Arriaga, I. P. "From distribution networks to smart distribution systems: Rethinking the regulation of European electricity DSOs", Utilities Policy, 31, pp. 229-237, 2014. https://doi.org/10.1016/j.jup.2014.03.007

[4] PeçasLopes, J. A., Hatziargyriou, N., Mutale, J., Djapig R. Jenkins, N. "Integrating distributed generation into electric systems: A review of drivers, challenges and opportunit Electric power systems research, 77(9), pp. 1189-1203, 2007. https://doi.org/10.1016/j.epsr.2006.08.016

[5] Gazijahani, F. S., Salehi, J. "Integrated scheduling for optimal operation of mid estimate method", International Jor Energy Systems, 99, pp. 481https://doi.org/10.1016/j.ijep

[6] Department of Energy
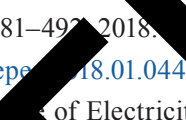
of Electricity D $\mathrm{D}$ and Energy Reliability "Summar
Chicago, Illinois, 20.

[7] Fathima, A. $y$ alanisamy, K. "OA zation in microgrids with hybrid en systems-A review", R able and Sustainable Energ htt oi.org/10.1 $431-446,2015$.

[8] Arefit A. amed, Y. A-P., EL-Fouly, T. H. M. "Optimum icrogrid for Enhan Reliability and Supply-Security", Transad on Sm arid, 4(3), pp. 1567-1575, 2013.

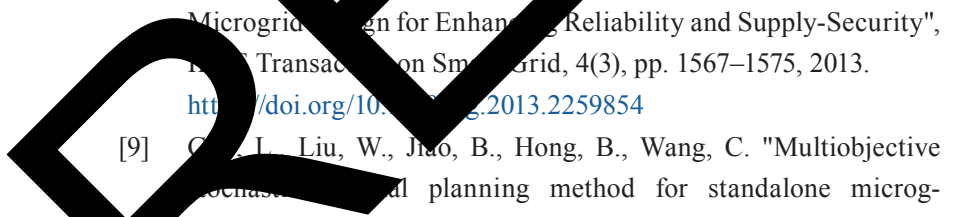
rid system", IET Generation Transmission Distribution, 8(7), pp. $1263-1273,2014$

\section{//doi.org/10.1049/iet-gtd.2013.0541}

[10] Whng, Z., Chen, B., Wang, J., Chen, C. "Networked Microgrids for Self-Healing Power Systems", IEEE Transaction on Smart Grid, 7(1), pp. 1-10, 2015.

https://doi.org/10.1109/tsg.2015.2427513 on the microgrids owners' policies. Since the proposed method is comprehensive and considers the system parameters uncertainties, it is an effective tool to design future distribution networks as interconnected microgridom ing into account system uncertainties.

[11] Wang, Z., Wang, J. "Self-Heal Based on sectionalization in Power System, 30(6), pp. 3

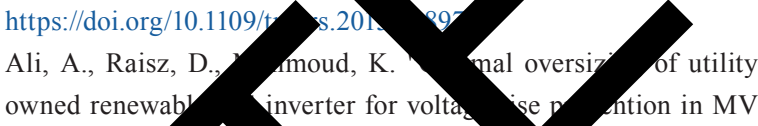
[12] Ali, A., Raisz, D., moud, K. mal oversiz of utility distribution ans in $\mathrm{MV}$ Energy Systums, 105, pp. $\quad$ 513, 2019. [13] hahramirad, S., Bhidehpour, M. "Microgrid 0(5), pp. 2417-242 015. Ltps://doi.org/10.110 pwrs.2014.2361094
Lidehpour, M., Alabdulwahab, A., A-Turki,
IEEE Mrame X., Smart Grid, 8(4), pp. 1771-1781, 2016.

as://doi.org/10.1109/tsg.2015.2508058

Alu., Yasser, I. M., El-Fouly, T. "Supply-adequacy-based optimal construction of microgrids in smart distribution systems", IEEE Transaction on Smart Grid, 3(3), pp. 1491-1502, 2012.

https://doi.org/10.1109/tsg.2012.2198246

] Arefifar, A., Yasser, I. M., El-Fouly, T. "DG mix, reactive sources and energy storage units for optimizing microgrid reliability and supply security", IEEE Transaction on Smart Grid, 5(4), pp. 1835-1844, 2014.

https://doi.org/10.1109/tsg.2014.2307919

[17] Arefifar, A., Yasser, I. M., El-Fouly, T. "Optimized multiple microgrid-based clustering of active distribution systems considering communication and control requirements", IEEE Transactions on Industrial Electronics, 62(2), pp. 711-723, 2015.

https://doi.org/10.1109/tie.2014.2352212

[18] Sedighizadeh, M., Esmaili, M., Jamshidi, A., Ghaderi, M. H. "Stochastic multi-objective economic-environmental energy and reserve scheduling of microgrids considering battery energy storage system", International Journal of Electrical Power \& Energy Systems, 106, pp. 1-16, 2019.

https://doi.org/10.1016/j.ijepes.2018.09.037

[19] Su, W., Wang, J., Roh, J. "Stochastic energy scheduling in microgrid with intermittent renewable energy resources", IEEE Transaction on Smart Grid, 5(4), pp. 1876-1883, 2014. https://doi.org/10.1109/tsg.2013.2280645

[20] Aien, M., Fotuhi-Firuzabad, M., Aminifar, F. "Probabilistic optimal power Flow in correlated hybrid wind-photovoltaic power systems", IEEE Transaction on Smart Grid, 5(1), pp. 130-138, 2014. https://doi.org/10.1109/tsg.2013.2293352 
[21] Moradi, M. H., Eskandari, M., Hosseinian, S. M. "Operational Strategy optimization in an optimal sized smart microgrid", IEEE Transaction on Smart Grid, 6(3), pp. 1087-1095, 2015. https://doi.org/10.1109/tsg.2014.2349795

[22] Soroudi, A., Caire, R., Hadjsaid, N., Ehsan, M. "Probabilistic dynamic multi-objective model for renewable and non-renewable distributed generation planning", IET Generation, Transmission, Distribution, 5(11), pp. 1173-1182, 2011. https://doi.org/10.1049/iet-gtd.2011.0173

[23] Pineda, S., Conejo, A. "Scenario reduction for risk-averse electricity trading", IET Generation, Transmission, Distribution, 4(6), pp. 694-705, 2010. https://doi.org/10.1049/iet-gtd.2009.0376

[24] Abido, M. "Environmental/economic power dispatch using multiobjective evolutionary algorithms", IEEE Transactions on Power Systems, 18(4), pp. 1529-1537, 2003. https://doi.org/10.1109/tpwrs.2003.818693

[25] Bompard, E., Carpaneto, E., Chicco, G., Napoli, R. "Convergence of the backward/forward sweep method for the load-flow analysis of radial distribution systems", International Journal of Electrical Power \& Energy Systems, 22(7), pp. 521-530, 2000. https://doi.org/10.1016/s0142-0615(00)00009-0

[26] Rajabioun, R. "Cuckoo Optimization Algorithm", Applied Soft Computing, 11(8), pp. 5508-5518, 2011. https://doi.org/10.1016/j.asoc.2011.05.008
[27] Ameli, A., Bahrami, S., Khazaeli, F., Haghi-fam, M. R. "A multiobjective particle swarm optimization for sizing and placement of DGs from DG owner's and distribution company's viewpoints", IEEE Transactions on Power Delivery, 29(4), pp. 1831-1840, 2014. https://doi.org/10.1109/tpwrd.2014.2300845

[28] Gazijahani, F. S., Salehi, J. "Stochastic multi for optimal dynamic planning of inter IET Renewable Power Generation, 11(14), https://doi.org/10.1049/iet-rpg.2017

[29] Gazijahani, F. S., Salehi, J. "Op based planning of microgric on Industrial Informatics https://doi.org/10.11

[30] Gazijahani, F.

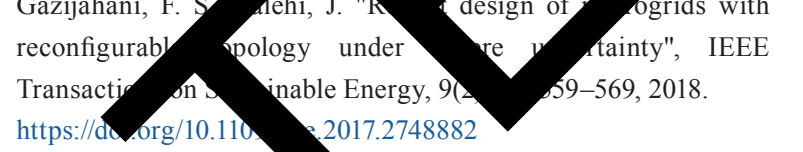

[31] Liu. G., Xu, Y., Tomson "Bidding strategy for microgrid in market based on stochastic/robust optimization", IEEE Transaction on Smart Gru, 7(1), pp. 227-237, 2016. https://doi.org/ $09 /$ tsg.2015.2476669

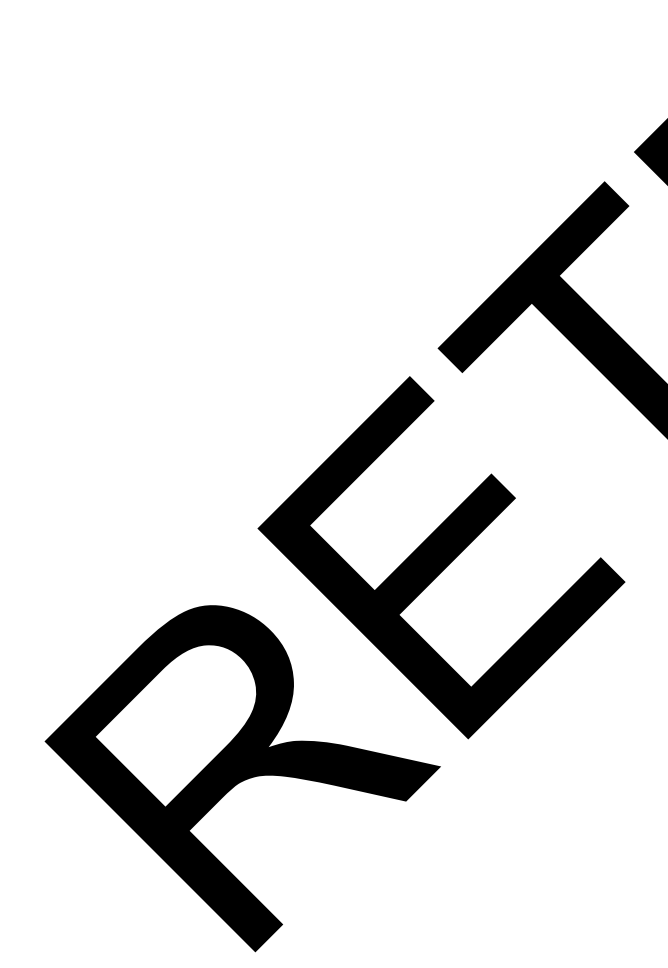

